DOI: https://doi.org/10.46296/rc.v4i8edesp.0032

\title{
Comunicación en Línea en la educación sincrónica y asincrónica en el pre-universitario
}

\section{Online communication in synchronous and asynchronous education in pre-university}

\author{
Meza-Intriago Felisa Hermelinda \\ Maestría en Comunicación - Mención Comunicación Digital. \\ Universidad Laica Eloy Alfaro de Manabí, ULEAM. Manta, Ecuador. \\ ORCID ID: https://orcid.org/0000-0002-8145-5159 \\ felisa.meza@uleam.edu.ec \\ Vásquez-Giler Maritza
}

Dirección de Posgrado, Cooperación y Relaciones Internacionales de la Universidad Laica Eloy Alfaro de Manabí, ULEAM. Manta, Ecuador. maritza.vasquez@uleam.edu.ec

\begin{abstract}
RESUMEN
Estos últimos años han sido muy determinante para la sociedad, a raíz de la declaración de la pandemia Covid-19, por parte de la OMS, muchas actividades se trasladaron a espacios virtuales, una de ellas es la educación, todo lo curricular se tuvo que replantear para lograr cumplir con el proceso de enseñanza aprendizaje; las instituciones de educación superior al igual que otras instituciones se vieron en necesidad de desarrollar de manera urgente, en los docentes y estudiantes, competencias digitales en las diferentes plataformas virtuales, de esto surgieron un sinnúmero de capacitaciones que les permitieran a ellos, desenvolverse en el mundo virtual enfocado en la educación. La comunicación en línea mediante las plataformas virtuales ha sido la herramienta de sostenibilidad para dinamizar la educación en tiempo de crisis sanitaria; en tal virtud surgió la interrogante ¿cómo la comunicación sincrónica y asincrónica influye en los resultados de enseñanza aprendizaje de los involucrados en el preuniversitario de la Universidad Laica Eloy Alfaro de Manabí durante el periodo 2020-2? Para este estudio se aplicó una metodología de enfoque mixto, donde lo cuantitativo sirvió en la recopilación de datos de fuente primaria, para a través del método cualitativo realizar un análisis descriptivo de la investigación planteada. Los resultados obtenidos llevaron a establecer que la educación virtual en el preuniversitario, la Uleam empleó las plataformas Moodle, Microsoft 365 y Zoom, donde docentes y estudiantes desarrollaron competencias digitales para interactuar en este proceso, no obstante, el nivel de dominio con la que utilizaban estos recursos didácticos digitales se ubica entre un nivel medio-alto, esta forma de educación y el manejo de herramientas digitales son muy necesarias e importantes para lograr un aprendizaje significativo, es por aquello que muchos estudiantes exteriorizan que los docentes deben prepararse más para que sean la guía de sus educandos.
\end{abstract}

Palabras claves: Comunicación en línea, educación sincrónica y asincrónica, plataformas virtuales, recursos didácticos digitales, enseñanza-aprendizaje virtual.

Información del manuscrito:

Fecha de recepción: 06 de septiembre de 2021.

Fecha de aceptación: 05 de octubre de 2021.

Fecha de publicación: 09 de noviembre de 2021. 


\begin{abstract}
These last years have been very decisive for society, following the declaration of the Covid-19 pandemic, by the WHO, many activities were transferred to virtual spaces, one of them is education. Everything in the curriculum had to be rethought in order to comply with the teachinglearning process. Universities, as well as other institutions, were in need of urgently developing, in teachers and students, digital skills in the different virtual platforms, from this arose a number of trainings that allowed them to develop in the virtual world focused on education. Online communication through virtual platforms has been the sustainability tool to boost education in times of health crisis; as a result, the question arose, how does synchronous and asynchronous communication influence the teaching-learning results of the members in the pre-university of the "Universidad Laica Eloy Alfaro de Manabi" during the period 2020-2? In order to carry out a descriptive analysis of the proposed research through the qualitative method. To carry out virtual education in pre-university, ULEAM used the Moodle, Microsoft 365 and Zoom platforms, where teachers and students developed digital skills to interact in this process, however, the level of mastery with which they used these digital teaching resources is located between a medium-high level, this form of education and the use of digital tools are very necessary and important to achieve meaningful learning, it is because of what many students express that teachers must prepare more to be the guide of their students.
\end{abstract}

Keywords: Online communication, synchronous and asynchronous education, virtual platforms, digital teaching resources, virtual teaching-learning.

\title{
1. INTRODUCCIÓN
}

La sociedad en general se ha tenido que adaptar a las nuevas herramientas tecnológicas para establecer comunicaciones bidireccionales que les permitan llevar a cabo una constante interacción, estos avances tecnológicos han tenido su mayor importancia y necesidad en estos tiempos de pandemia, donde muchas actividades tuvieron que emigrar de lo tradicional a lo virtual.

La presente investigación abordó temas como el de la comunicación en línea aplicada en la educación sincrónica y asincrónica del preuniversitario de la Universidad Laica Eloy Alfaro de Manabí, (en adelante Uleam), la actual forma de enseñanza - aprendizaje es aquella que se ha da de manera virtual por medio de las diferentes plataformas virtuales, en este sentido se dará un enfoque desde la educación, que tanto docentes como estudiantes han tenido que adecuar la forma de brindar y recibir los conocimientos, esta forma de comunicación virtual permite que los individuos se enlacen, informen, analicen, cuestionen, debatan y compartan temas de interés, en cualquier lugar y en cualquier momento, lo que ha sido denominado como "auto comunicación de masas" (Castells, 2009). Conforme a lo mencionado, esta comunicación entrelaza a los involucrados para 
coordinar acciones y cumplir con actividades que se llevan a cabo en el ámbito educativo, que sin lugar duda no hubiese sido posible sin la evolución de la tecnología, es gracias a ello que existen plataformas virtuales que se ajustan a esta necesidad.

En este contexto de la educación en línea, surge la comunicación sincrónica y asincrónica. Lamí, (2016) enfatiza que la sincronía coincide en tiempo y espacio en una determinada plataforma, mientras que la asincronía es en parte lo contrario de ésta y se da en distintos momentos; y es a través de ello que la educación ha ido tomando mayor fuerza gracias a las bondades que ofrece el progreso tecnológico; la fusión de estas dos aristas ha permitido conseguir grandes desafíos como la formación continua y profesionalizante en línea. La oportunidad que ofrecen estos nuevos escenarios virtuales aparece como un gran soporte en la educación frente a la pandemia del 2020, donde las universidades se vieron en la necesidad de replantear lo curricular en cuanto a la forma y la utilización de los medios para llevar a cabo el proceso de enseñanza aprendizaje.

En este sentido, las IES (Instituciones de educación superior) específicamente la Uleam, conjuntamente con sus involucrados se vieron en la necesidad de innovar e implementar nuevas formas de distribuir el conocimiento, lo que permitió desarrollar nuevas competencias y destrezas tanto a docentes como a estudiantes, quienes acogieron las distintas herramientas digitales que facilitaban el avance de este proceso académico, es así que la educación en línea como lo dice Caldeiro 2014, "se caracteriza por generar espacios alternativos mediados por tecnología digital en donde se promueve la construcción colaborativa del conocimiento", (p.16). bajo esta determinación y entendiendo que muchos docentes y estudiantes no estaban acostumbrados a esta forma de enseñar y aprender, nace la interrogante ¿Cómo influye la comunicación digital en la educación sincrónica y asincrónica en el preuniversitario de la Uleam?, que determinarán mediante la aplicación de la metodología.

Por lo tanto, en este estudio se pretende determinar cómo la comunicación en línea en el pre-universitario de la Uleam influye en los resultados de la enseñanza 
aprendizaje en el periodo 2020-2, tomando como acciones el identificar qué plataformas virtuales se emplearon para llevar a cabo el proceso; así como también, establecer el dominio de las plataformas digitales y determinar la importancia que tuvieron los recursos didácticos digitales en docentes y alumnos para lograr un aprendizaje significativo en los estudiantes del pre-universitario en el periodo 2020-2.

\section{- La comunicación en línea sincrónica y asincrónica}

La comunicación en línea o comunicación online, es el intercambio de mensajes a través de las diferentes plataformas que ofrece internet, con las herramientas necesarias los usuarios de dichas plataformas pueden compartir todo tipo de información en el ámbito digital (Vite, 2020).

Existe muchas maneras de comunicarse gracias al internet, los usuarios pueden entablar conversaciones en línea con amigos, colegas, familiares, incluso con desconocidos, hoy en día existen diversas maneras de llegar a las personas que tradicionalmente eran difíciles de contactar, en este sentido la tecnología juega un papel importante, pues cada vez que avanza, las formas de comunicarse se vuelven más ágiles.

La comunicación en línea está intrínsecamente relacionada con la educación, ya que es una forma plena para desarrollarse dentro de ese contexto a través de las diferentes plataformas web, de esta manera el aprendizaje humano está relacionada con la educación. Los estudios recientes sobre el futuro de la educación indican que la enseñanza-aprendizaje en línea está siendo cada vez más adoptada por instituciones de alto prestigio académico en todo el mundo (Guerrero et. al. 2020).

Velásquez et. al. (2018), establece 9 formas de comunicación en línea por diferentes plataformas web y estas se pueden dar de manera sincrónica o asincrónica dependiendo de la intencionalidad de la persona: 1.- Comunicación vía correo electrónico. 2.- Mensajería instantánea. 3.- Salas de conversación virtual. 4.- Redes sociales. 5.- Foros virtuales. 6.- Blogs y páginas web. 7.Videoconferencias y audioconferencias. 8.- Llamadas telefónicas en línea. 9.Aplicaciones web. 
Quizás en el presente haya otras maneras de comunicarse por internet, según Del Prete \& Cabero (2019), en la actualidad las plataformas virtuales, representan un instrumento importante para la comunicación en línea, sobre todo dentro del ámbito educativo, debido a que tienen la capacidad de proveer a los estudiantes un aprendizaje personalizado.

Sánchez, y García (2008), a través una investigación sobre Práctica del consumo audiovisual en internet en estudiantes universitarios de la ciudad de Medellín, determinaron tres metodologías de enseñanza-aprendizaje: que es el sincrónico, asincrónico y a la vez el método B-learning (método combinado). Es así como la comunicación y los estudios en línea se pueden realizar de las tres maneras, entendiéndose que la educación sincrónica es aquella que permite el encuentro entre docente y estudiante en un tiempo determinado, utilizando la misma plataforma virtual; y a su vez la educación asincrónica se da sin la figura de un profesor, es decir no se necesita la presencia del tutor para poder realizar cualquier trabajo educativo (Del Prete \& Cabero, 2019).

El método B-learning es un proceso de enseñanza y aprendizaje mixto, es decir que integra a la modalidad presencial con lo virtual en su misma forma para desarrollarse, donde la interacción pasaría a ser semipresencial, es decir, se combina el trabajo presencial y el trabajo en línea, acompañado de las TICS para desarrollar los procesos de comunicación (Sánchez y García, 2008).

El uso cotidiano de herramientas, así como el empleo de las Tecnologías de la Información y la Comunicación es cada vez más común en el ámbito educativo, más aún cuando se trabaja en el nivel de Educación Superior, y es un recurso fundamental en la modalidad virtual, en donde es posible establecer comunicación tanto de manera sincrónica como asincrónica.

- Principales plataformas virtuales para el proceso de enseñanzaaprendizaje

Las TICS se han convertido en un conjunto de herramientas que aportan diferentes elementos para mejorar el proceso de enseñanza; misma que busca desarrollar escenarios para mejorar la interacción entre el docente y el 
estudiante, siendo un elemento que aporta significativamente al proceso de formación profesional (Cabrera \& Dinely, 2003).

Las plataformas virtuales son programas desarrollados en softwares que permiten a la computadora realizar determinadas tareas en internet, para mejorar la comunicación y el aprendizaje individual y colectivo.

Bajo este contexto, las plataformas virtuales son utilizadas de diferentes maneras con la finalidad de aportar al desarrollo de entornos virtuales para maximizar el potencial de los usuarios, abriendo un abanico de posibilidades para desarrollar habilidades y competencias tecnológicas que permitan el uso correcto de la tecnología aplicados a ambientes educativos (Prete \& Almenara, 2020).

Según, Corona (2012), el internet se ha consolidado como el más importante desarrollo tecnológico que integra adelantos fundamentales para la comunicación. Por esta razón, Gonzales (2011), establece que los usuarios en internet tienen la necesidad de satisfacer una carencia, es por este motivo que deciden utilizar las diferentes plataformas virtuales.

Salvador (2005), plantea que una plataforma virtual flexible es aquella que permite adaptarse a las necesidades de los alumnos y profesores, es decir acoplarse a las distintas herramientas que ésta trae en su interfaz y que a la vez presenta funcionalidad amigable y fácil de utilizar.

La plataforma educativa es una herramienta indispensable para la interacción y comunicación de los cursos que se imparten en modalidad a distancia, sin embargo, al inicio puede resultar complicado adaptarse al uso de esta herramienta porque la mayoría de los alumnos están acostumbrados al modelo tradicional (Guerrero et. al. 2020).

Las plataformas virtuales se fueron adaptando al proceso educativo sobre todo en el año 2020, ya que en este tiempo por razones de pandemia se tuvo que adaptar el proceso de enseñanza en el entorno virtual. Según Miguel Román (2020), El cambio de la modalidad presencial a lo virtual, en algunas instituciones no alteró mayormente el proceso de enseñanza - aprendizaje, ya que muchos docentes y estudiantes ya estaban familiarizados con este avance digital. 
Los autores Guerrero, Vite y Feijoo, (2020), quienes realizaron una investigación sobre el Uso de la Tecnología de Información y Comuni-cación y las Tecnologías de Aprendizaje y Conocimiento en tiempos de Covid-19 en la educación superior, determinaron que de manera general las plataformas que más se utilizan son: TIC, Moodle, Blackboard, Edmodo, Teams, TAC, Tareas, Foros, Wikis, Cuestionarios, Evaluación; las cuales han sido consideradas como mediadores para la entrega de contenidos y actividades académicas que el docente planifica.

Luego de la investigación realizada determinaron que "la tecnología de información y comunicación (TIC) y la tecnología de aprendizaje y conocimiento (TAC), son herramientas ne $\neg$ cesarias en estos tiempos de confinamiento y emergenᄀcia sanitaria Covid-19" (Guerrero et al. 2020, p344), por lo que es importante y necesario potenciar a los docentes y alumnos de las instituciones.

La Uleam, en el preuniversitario, durante el periodo 2020-2 ante la crisis sanitaria presentada a nivel mundial, se vio en la necesidad de emplear varias plataformas que han sido consideradas por los autores anteriores, y otras que han sido determinante para el proceso de enseñanza aprendizaje tales como se describe a continuación:

Moodle.- Es una plataforma para la gestión de cursos en línea, (Martínez 2007:2), "es la más potente herramienta con la que contamos los docentes en este momento para poder crear y gestionar nuestro curso a través de la red"; el uso de esta plataforma permite trabajar de manera asincrónica, a través de esta aula virtual el estudiante puede realizar un sinnúmero de actividades como: subir tareas o trabajos asignados por el docente, mientras que los docentes pueden gestionar cursos, temas y/o contenidos de forma sencilla, en los que podemos incluir gran variedad de actividades y hacer un seguimiento exhaustivo del trabajo de los estudiantes, también permite la comunicación a distancia mediante foros, correo y chat, favoreciendo así el aprendizaje cooperativo. Este se basa en la pedagogía social constructivista (colaboración, actividades, reflexión, etc.).

La navegación es accesible, confiable y estable, así como ligera, sencilla y compatible con distintos navegadores Web. También permiten al administrador del sitio personalizar colores, tipos de letra a su gusto o necesidad. Estas plantillas son fáciles de modificar y ampliar; además, contribuye para que los 
educadores creen espacios virtuales de aprendizaje en los que desarrollar cursos on-line o utilizarlos de apoyo como complemento a la enseñanza tradicional (González, 2014).

Microsoft 365.- también conocido como Office 365, es una herramienta creada por Microsoft, Cabrera, (2020) esta plataforma es de uso en la educación virtual, la cual permite trabajar de manera sincrónica y asincrónica; entre las bondades que se encuentran están: crear y compartir documentos en línea entre distintos usuarios, además por medio de sus aplicaciones como Word, Excel, PowerPoint y OneNote, Streams, entre otros. Se pude llevar una planificación muy favorable con los involucrados, que va desde agendar una reunión, notificar, recibir tareas, mantener reuniones y grabarlas, y una variedad de alternativas más. Para lograr esta conectividad, solo se necesita tener acceso a internet y disponer de un usuario y una contraseña que se es dado por la institución donde pertenece.

Dentro de Microsoft 365, resalta la aplicación Microsoft Teams la cual es una plataforma de comunicación y colaboración unificada a través de chats y reuniones de video entre usuarios Cabrera, (2020).

Según, Corona (2012), el internet se ha posesionado como el más importante desarrollo tecnológico que integra adelantos fundamentales para la comunicación en línea, a través del surgimiento de este, son cada vez más los beneficios se encuentran gracias al desarrollado tecnológico. Por esta razón, Gonzales (2011) establece que gracias a internet se puede satisfacer necesidades de todo tipo, desde consultas básicas hasta las más complejas, convirtiéndose así en una herramienta actual donde los usuarios tienen la necesidad de satisfacer una carencia, es por este motivo que deciden iniciar una búsqueda de información o utilizar cualquiera plataforma en línea.

Zoom.- Es una aplicación de videoconferencia que incluye un chat de mensajería en tiempo real e intercambio de contenido información de manera fácil y sencilla, según Morales y Puentes, (2019), a través de esta plataforma se aumenta la interactividad de los participantes y de esta manera incrementa el aprendizaje. Con esta aplicación pueden crearse reuniones de 100 usuarios de forma gratuita. Zoom ofrece un entorno virtual y seguro, dada su reciente popularidad Zoom está proporcionando parches de seguridad, y pretende seguir mejorando la 
aplicación, aunque si reúna mínimos requisitos de seguridad en la configuración y se puede tener en cuenta como un buen aliado durante la crisis del Covid-19 en los escenarios de teletrabajos y telestudios.

De acuerdo con el párrafo anterior se considera asumible el riesgo de usar Zoom para reuniones del ámbito educativo que favorezcan al proceso de enseñanzaaprendizaje, clases escolares y situaciones fuera de la oficina con distintos fines.

Los sitios web se utilizan frecuentemente para el manejo y divulgación de información, pero sobre todo como medio de comunicación entre personas y son accesibles 24 horas al día, siete días a la semana a través de la internet (Calvo, 2015, p. 2).

\section{- Recursos didácticos en el aprendizaje significativo}

Un aprendizaje es significativo cuando el estudiante logra adquirir los conocimientos pertinentes y con ellos desarrollar habilidades y competencias que le permita relacionar de manera óptima y valiosa lo comprendido, de tal manera que esos conocimientos sean aplicados para integrar lo teórico con lo práctico; por lo tanto, la estructura cognitiva bien concebida, le permite al estudiante extrapolar y adquirir aptitudes propias para realizar un producto necesario y significativo.

En este sentido, es preciso mencionar que en los procesos educativos es importante considerar lo que el estudiante ya sabe, de tal manera que establezca una relación con aquello que desea aprender, este proceso tiene lugar si el alumno tiene en su estructura cognitiva previa conceptos o ideas de los temas que se van a tratar.

En las últimas décadas la educación ha evolucionado significativamente en función de incorporar recursos y herramientas tecnológicas de la información y la comu $n$ nicación; sin embargo, la situación actual a nivel global ha obligado a tomar acciones inmediatas. Por ello, las instituciones de educación superior tienen la necesidad de garantizar la profesionalización docente como vía fundamental para incrementar la calidad del proceso de formación del profesional universitario en el reto actual. Ahora más que nunca, se requiere de 
profesionales capa $\neg$ citados para enfrentar la transformación de la sociedad (Guerrero et. al. 2020).

Las plataformas virtuales educativas, son entornos que ayudan al aprendizaje significativo para utilizarlas en el proceso de enseñanza-aprendizaje online, es necesario tener en consideración un programa que pueda integrar las diversas y principales herramientas que ofrece el internet.

Salvador (2005), plantea que una plataforma virtual es aquella que permite adaptar las necesidades de profesores y alumnos, es decir acoplar las distintitas herramientas que ofrece con un interfaz amigable, fácil de utilizar y con una navegabilidad clara y homogénea en todas sus partes.

La conectividad y la interacción que se genere dentro de los espacios virtuales es de eje medular en el proceso de construcción del conocimiento, puesto que se ponen en juego un conjunto de aspectos que se relacionan entre sí, desde la misma interacción entre usuarios en un determinado sitio contribuye al aprendizaje significativo (Ruiz, et al., 2012), lo que hace necesario incluir en las TIC, las CVA (Comunidades Virtuales de Aprendizaje) adopten costumbres y mecanismos de transmisión de información desde punto de vista pedagógico que impulsen las nuevas formas de aprender.

Bajo este contexto, adquirir nuevas competencias digitales es esencial para la responder a modalidad de la educación actual, y saber manejar y/o construir recursos didácticos digitales permiten que docentes y estudiantes estén a la par y logren una educación de calidad; (Ruiz, et al., 2012, p5.), manifiesta que "el aprendizaje significativo en los ambientes virtuales debe ser un proceso de búsqueda de significados, conocimientos sociales que trascienden a escenarios más complejos, que son aplicados y transferidos a realidades profesionales 0 personales de la cotidianidad".

En este sentido el que docentes y estudiantes aprendan de manera integral mediante video conferencia, foros, talleres interactivos, laboratorios virtuales, ilustradores de gráficos, etc. potencien las competencias digitales para construir un proceso de enseñanza que fortalezca los conocimientos de los educandos, para lo cual se debe tomar en consideración nuevas acciones en la educación continua (Guerrero, Vite y Feijoo, 2020). En tan virtud que, logradas en absolutos 
estas destrezas digitales los docentes puedan incrementar la calidad de la educación en línea.

\section{METODOLOGÍA}

La investigación se la realizó dentro de la Uleam tomando como tiempo de estudio el periodo 2020-2 en el contexto del preuniversitario, se aplicó una metodología de cohorte mixto cuanti y cualitativo, por lo que dan una complementariedad al estudio realizado, ambos métodos son muy aplicable en las ciencias sociales lo que permite lograr una investigación flexible, válida y confiable (Cadena, et al 2017).

La búsqueda y selección de información mediante la revisión de la literatura, fue muy determinante para establecer una investigación de tipo cualitativo, lo que permitió fundamentar teóricamente las variables y los objetivos planteados en la investigación, así como la aplicación de entrevista a experto para luego mediante un análisis descriptivo, determinar aspectos esenciales en cuanto a la comunicación en línea sincrónica y asincrónica frente a la educación en la Uleam en el preuniversitario.

Así pues, también se consideró el enfoque cuantitativo, por lo que se aplicaron encuesta tanto a docentes como estudiantes. Para obtención de información de los docentes, se consideró el 100\% es decir el total de los involucrados, en este sentidos la muestra fue 33 personas, quienes formaron parte del proceso de enseñanza del periodo académico; para determinar la muestra de los estudiantes se utilizó la aplicación surveymonkey.com donde se ubicó el universo estudiantil, tomando en cuenta un $95 \%$ nivel de confianza, y solo el $5 \%$ como límite de error muestral, dicho universo fue de 3200 estudiantes, de la cual la aplicación determinó aplicarse la encuesta a 344 estudiantes del preuniversitario del 2020-2, los datos obtenidos de esta aplicación permitieron recoger información muy valiosas que ayudaron a entender mejor la influencia de la comunicación en línea en la educación de los preuniversitarios. 
Además de las fuentes primarias como las descritas anteriormente, también fueron de mucha valía las fuentes secundarias obtenidas desde los repositorios científicos como: Scielo, Redalyc, Google académico, Dialnet, entre otros.

Una vez obtenida la información requerida, se realizó un análisis descriptivo sobre el uso de las plataformas y el impacto que ha generado en el aprendizaje significativos.

\section{ANÁLISIS DE LOS RESULTADOS}

La entrevista realizada al Ing. Jacinto Flores, experto en informática y funcionario de la Uleam en la DIIT (dirección de informática e innovación tecnológica) a quien se le preguntó cuales habían sido las plataformas utilizadas para llevar acabo la enseñanza y aprendizaje en los preuniversitarios de la Uleam, exteriorizó que fueron tres las plataformas utilizadas: Moodle y Teams más la plataforma Zoom; siendo de mayor uso para el tema de Moodle https://moodledanu.uleam.edu.ec/ para las actividades asincrónica y Zoom https://salavirtual.cedia.org.ec/ para las actividades sincrónica.

El ing. Flores, indicó que en la transición de esta nueva modalidad de enseñanza se tomaron varias acciones que iba desde capacitaciones permanentes a los involucrados hasta las frecuentes actualizaciones en las plataformas para mejorar el servicio a los estudiantes, también enfatizó que las plataformas virtuales siempre han estado operativas en la nube, y que si bien había ciertas incidencias eran porque la conexión del estudiante en mucho caso, no era de buena calidad.

Las encuestas aplicadas a los 33 docentes que impartieron clases y a los 344 estudiantes que la recibieron, durante el periodo 2020-2 en el preuniversitario de la Universidad Laica Eloy Alfaro de Manabí, arrojaron resultados muy determinantes en la validación y sostenibilidad del análisis descriptivo en referencia a la comunicación en línea y su influencia en los resultados de la enseñanza - aprendizaje.

Es así que el interés de conocer qué plataformas virtuales habían empleado los docentes y estudiantes para llevar a cabo este proceso, ambas partes coincidieron con el $100 \%$ que la plataforma que utilizan para la asincronía es la 
plataforma Moodle; donde pudieron dar cumplimiento a las actividades académicas asignadas; así también coinciden que la plataforma virtual que emplean para llevar el proceso de manera sincrónica, es Zoom, donde los involucrados se encuentran en la misma plataforma y en un horario específico establecido.

Para establecer qué dominio tienen los docentes para enseñar a través de los recursos didácticos digitales, se logró identificar que existen un nivel alto - medio en la realización de sus propios recursos para el proceso educativo; mientras que, contrastando dicha información con los estudiantes, el porcentaje mayor que es del 53\%, manifestó que los docentes tienen un nivel medio en el manejo de estos recursos y por lo tanto los docentes sí necesitan capacitarse para mejorar en su enseñanza.

Se le consultó a los docentes, cuáles fueron las competencias tecnológicas desarrolladas y en cuáles tienen mayor dominio, para esto se les ubicó varias alternativas, obteniendo datos como: un 43\% manifestó el dominios en guías didácticas; $24 \%$ en la utilización de videos de YouTube; un 15\% en la realización de cuestionarios en líneas; entendiéndose que lo fuerte en ellos es la realización de guías metodológicas, puesto que este recurso les ayuda a orientar de mejor manera a sus estudiantes; sin embargo, también han logrados adquirir otras competencias en menor grado, en cuanto a la utilización de recursos en línea desde las plataformas Moodle y Zoom; por otro lado y en similar pregunta, los estudiantes indicaron con un $76 \%$ tener el dominio de subir sus tareas en las plataformas antes descritas.

En consecuencia, con los objetivos planteados también se realizaron las siguientes preguntas a los encuestados de las que se obtuvieron los siguientes resultados:

Tabla 1. ¿En qué nivel considera usted que la comunicación en línea mediante la utilización de recursos didácticos ha influido en el aprendizaje significativo de sus estudiantes?

\begin{tabular}{|c|c|c|}
\hline Variables & Docentes & Porcentaje \% \\
\hline Alto & 18 & $54.5 \%$ \\
\hline Medio & 15 & $45.5 \%$ \\
\hline Bajo & 0 & - \\
\hline Total & 33 & $100 \%$ \\
\hline
\end{tabular}


Para determinar si la comunicación en línea, mediante la utilización de recursos didácticos digitales influye en el aprendizaje significativo se recogieron datos muy importantes donde los docentes con el 54,5\% indicaron que en nivel de influencia es alto, sin embargo, es importante mencionar que el porcentaje restante manifestó que ha influido medianamente.

Tabla 2. ¿Cree Usted que la comunicación en línea mediante la utilización de recursos didácticos ha influido en el aprendizaje de los estudiantes, en qué manera?

\begin{tabular}{lll}
\hline \multicolumn{1}{c}{ Variables } & \multicolumn{1}{c}{ Estudiantes } & Porcentaje \% \\
\hline Muy significativo & 194 & $57,00 \%$ \\
Poco significativo & 141 & $41,5 \%$ \\
Nada significativo & 5 & $1,5 \%$ \\
\hline Total & $\mathbf{3 4 4}$ & $\mathbf{1 0 0 \%}$ \\
\hline
\end{tabular}

Así también, los estudiantes también coinciden con los docentes, y el 57\% manifestó que el aprendizaje es muy significativo; Sin embargo, otro grupo de estudiantes con el 41,5\% consideran que es poco significativo; el restante 1,5\% mencionó que es nada significativo.

Tabla 3. ¿Qué grado de dificultad ha encontrado Usted en el aprendizaje con la modalidad virtual?

\begin{tabular}{|c|c|c|}
\hline Variables & Estudiantes & Porcentaje \% \\
\hline Alto & 43 & $12,6 \%$ \\
\hline Medio & 230 & $67,3 \%$ \\
\hline Bajo & 70 & $20,1 \%$ \\
\hline Total & 344 & $100 \%$ \\
\hline
\end{tabular}

De acuerdo con lo consultado sobre qué nivel de dificultad habían encontrado para aprender, de los 344 estudiantes encuestados, 230, que equivale al $67,30 \%$ exteriorizó que las dificultades presentadas estuvieron en un nivel medio; mientras que el $12,6 \%$ expresó que encuentra un nivel alto de dificultad para aprender en la modalidad virtual: el restante $20,1 \%$ mencionó que el nivel de dificultad es bajo. 
Tabla 4. ¿Cómo considera Usted que ha sido el estudio de manera virtual?

\begin{tabular}{llr}
\hline \multicolumn{1}{c}{ Variables } & Estudiantes & Porcentaje \% \\
\hline Muy favorable & 150 & $44,0 \%$ \\
Poco favorable & 179 & $52.5 \%$ \\
Nada favorable & 12 & $3.5 \%$ \\
\hline Total & $\mathbf{3 4 4}$ & $\mathbf{1 0 0 \%}$ \\
\hline
\end{tabular}

Se les consultó a los estudiantes si la educación virtual había sido favorable un $52,5 \%$ manifestó que la educación había sido poco favorable; No obstante, el $44 \%$ mencionó que era muy favorable; y solo el 3,5\% consideró que no era favorable.

Tabla 5. ¿Cree Usted que a los docentes les falta desarrollar competencias digitales para enseñar de mejor manera a los estudiantes?

\begin{tabular}{|c|c|c|}
\hline Variables & Estudiantes & Porcentaje \% \\
\hline $\mathrm{Si}$ & 192 & $56,3 \%$ \\
\hline No & 149 & $43,7 \%$ \\
\hline Total & $\overline{344}$ & $100 \%$ \\
\hline
\end{tabular}

Esta pregunta es muy fundamental para esta investigación porque arrojaron resultados muy determinantes, donde el $56 \%$ de los encuestados indican que a los docentes les hace falta desarrollar competencias digitales enfocado en la enseñanza; mientras que el porcentaje restante indicó que no les hace falta.

\section{DISCUSIÓN}

En el contexto de esta investigación, la autora se propuso determinar si la comunicación en línea en el pre-universitario de la Uleam influyó en los resultados de la enseñanza aprendizaje en el periodo 2020-2 y de los resultados obtenidos aplicados a docentes y estudiantes determinaron mayoritariamente que la comunicación en línea ha influido de manera alta y significativamente en el aprendizaje, mediante la utilización de las herramientas tecnológicas, lo cual se confirma con la teoría de Del Prete \& Cabero (2019), en la que dicen que en la actualidad las plataformas virtuales, representan un instrumento importante para la comunicación en línea, sobre todo dentro del ámbito educativo, debido a 
que tienen la capacidad de proveer a los estudiantes un aprendizaje personalizado.

Desde la concepción de Ruiz, et al., (2012), para que haya un aprendizaje significativo en los ambientes virtuales, tanto docentes como estudiantes deben estar permanentemente en la búsqueda de significados y de nuevos conocimientos que trasciendan a niveles más altos y a la par con la tecnología; frente a los resultados obtenidos, se determinó que los docentes tiene un nivel alto-medio en la realización y utilización de los recursos tecnológico; así también los estudiantes indicaron que si bien es cierto, los docentes saben impartir las cátedra de manera virtual, pero que aún falta que desarrollen otras competencias para que la enseñanza fluya; En tal virtud, y, por la premura en que la educación en la sociedad por efecto de la pandemia se volcó a los escenarios virtuales, los docentes han logrado adquirir ciertas competencias básicas que les ha permitido interactuar con el estudiante; sin embargo, las plataformas ofrecen un sinnúmero de bondades que fortalecen este sistema educación solo que hay que descubrirlas y aprenderlas; por eso Guerrero, Vite y Feijoo, (2020), propone considerar nuevas acciones y capacitación continua, para alcanzar los estándares de calidad en la educación.

\section{CONCLUSIONES}

El estudio realizado ha sido de mucha valía, porque se ha comprobado el entrelazado de las múltiples formas digitales y la educación, de esta manera los objetivos planteados a través de la literatura revisada y de los resultados obtenidos de las técnicas aplicadas han permitido llegar a las siguientes conclusiones:

La comunicación en línea en el pre-universitario de la Uleam influyó en los resultados de la enseñanza aprendizaje en el periodo 2020-2. Esta forma de comunicación digital mediante la sincronía y asincronía permitió que docentes y estudiantes mantuvieran una permanente interacción en el cumplimento de las actividades académica planificadas curricularmente. 
Las plataformas virtuales que se utilizaron en el pre univiersitario de la Uleam durante el periodo 2020-2 fueron muy importante para el desarrollo del proceso de enseñanza-aprendizaje, Moodle y Microsoft Office 365, facilitaron la planificación y realización de las actividades de manera asincrónica; mientras que la plataforma Zoom fue utilizada para la conectividad sincronía; ambas plataformas se complementaron entre sí durante en el periodo del tiempo estudiado.

El dominio que tienen los docentes del pre-universitario en cuanto al manejo de las plataformas virtuales y utilización de recursos didácticos digitales, se encuentra en un nivel medio-alto, criterio que coincidió en docentes y estudiantes; con esta información se hace necesario tomar en cuenta que el nivel medio, deja un grado de insatisfacción, porque denota que todavía faltan desarrollar competencias digitales enfocadas a la educación para tener mejores resultados en la enseñanza-aprendizaje.

La utilización de los recursos didácticos digitales en el preuniversitario fue muy importante en los resultados del aprendizaje, por lo que se recomienda a docentes y estudiantes seguir con el compromiso de desarrollar un amplio conocimiento dentro de los espacios virtuales, y adquirir competencias digitales enfocado a lo educativo, porque el avance tecnológico cada vez se posesiona con mayor fuerza y con innovaciones en la educación.

\section{REFERENCIAS}

Ayala Pezzutti, Rocío Janett, Laurente Cárdenas, Carlos Miguel, Escuza Mesías, César Daniel, Núñez Lira, Luis Alberto, \& Díaz Dumont, Jorge Rafael. (2020). Mundos virtuales y el aprendizaje inmersivo en educación superior. Propósitos y Representaciones, 8(1), e430. https://dx.doi.org/10.20511/pyr2020.v8n1.430

Cabrera Delgado, J. M. (2020). Plataformas para el aprendizaje en línea: La protección de datos en el ámbito educativo. Avances En Supervisión Educativa, (33). https://doi.org/10.23824/ase.v0i33.680

Cabrera \& Dinely (2003). DISCURSO DOCENTE EN EL AULA. Estudios Pedagógicos, (29),7-26. [fecha de Consulta 5 de Julio de 2021]. ISSN: 
0716-050X.

Disponible

en: https://www.redalyc.org/articulo.oa?id=173514130001

Caldeiro G, (2014), La comunicación en línea y el trabajo colaborativo mediado por tecnologías digitales, Astral Comunicación, 3(1), 13-30.

Calvo Guillén, G. (2015). Rediseño de un sitio web como sistema de información mediante la arquitectura de información: en busca del fortalecimiento de la comunicación. Revista e- Ciencias de la Información, 5 (1), 1-25.

Corona Rodríguez, J. (2012). La construcción social de internet: estrategias de uso y significación de la información. Paakat: Revista de Tecnología y Sociedad, (3), 1-14.

Del Prete, A., \& Cabero Almenara, J. (2019). Las plataformas de formación virtual: algunas variables que determinan su utilización. Apertura, 11(2), 138-153. https://doi.org/10.32870/ap.v11n2.1521

González, J. (2014). es.slideshare.net. Obtenido de Moodle: http://es.slideshare.net/josealexmas/moodle-36211437

González, M. (2011). La Web 2.0 en los sistemas de recuperación de información.

Biblios,

(44),

$35-40$.

http://scielo.sld.cu/scielo.php?script=sci_arttext\&pid=S1990-

$86442020000600338 \& \operatorname{lng}=$ es\&tlng=es

Jin, S. y C.M. Cho, (2015), Is ICT a new essential for national economic growth in an information society? Government Information Quarterly, 32(3), 253260.

Lamí, L., Pérez M. \& Rodríguez, M. (2016). Las herramientas de comunicación sincrónica y asincrónica en la clase presencial. Revista Conrado, 12(56), 84-89. Recuperado de http://conrado.ucf.edu.cu/

Martínez, I. (2008). Moodle, la plataforma para la enseñanza y organización escolar. Ikastorratza. e-Revista de didáctica, (2), 1-12. https://redined.educacion.gob.es/xmlui/handle/11162/201846

Mazzetti Latini, C. (2014). Una aproximación a las intimidades visibles en el escenario digital. Una Aproximación a Las Intimidades Visibles En El Escenario Digital, 5(0), 125-137. https://dialnet.unirioja.es/servlet/articulo?codigo=7219800

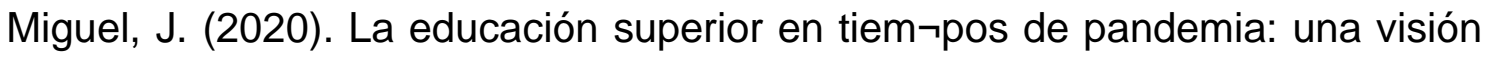
desde dentro del pro $\neg$ ceso formativo. Revista Latinoamericana de Estudios Educativos, 50, 13-40.

Morales, E. M., \& Puentes, Ú. (2019). El uso de la Herramienta Zoom y el aprendizaje colaborativo en un entorno virtual. Obtenido de Repositorio 
http://rai.uapa.edu.do:8080/xmlui/handle/123456789/544?show=full

Prete, A., \& Almenara, J. (2020). Las plataformas de formación virtual: algunas variables que determinan su utilización. Revista de Innovación Educativa, 11(2),138-153.

http://www.udgvirtual.udg.mx/apertura/index.php/apertura/article/view/15 21

Salvador, P. i G. (2005). Nuevos espacios y entornos de educación. Alicante: ECU.Sánchez, R y García. J (2008). Práctica del consumo audiovisual en internet en estudiantes universitarios de la ciudad de Medellín. (Tesis doctoral). Universidad Pontifica Bolivariana, Medellín.

Velásquez, O., Urresty, J., Miramá, V., Hernández, C. and Jojoa, P., (2018) Desempeño de un Sistema de Comunicación Digital Empleando Codificación de Línea. Revista Ingenierías USBMed, [online] (2), pp.1118.

Available

at: https://dialnet.unirioja.es/servlet/articulo?codigo $=6523323$

Véliz, M., y Gutiérrez, V. (2021). Modelos de enseñanza sobre buenas prácticas docentes en las aulas virtuales. Revista Apertura, 13(1), 150-165. Recuperado http://www.udgvirtual.udg.mx/apertura/index.php/apertura/article/view/19 $87 / 1276$

Veytia Bucheli, M. G. (2021). La estructura argumentativa en estudiantes de posgrado a partir de foros virtuales. Revista Conrado, 17(78), 234-239.

Vite, H. (2020). Estrategias tecnológicas y metodológicas para el desarrollo de clases online en instituciones educativas. Conrado, 16(75), 259-265. http://scielo.sld.cu/scielo.php?script=sci_arttext\&pid=S1990-

86442020000400259\&lng=es\&tlng=es 